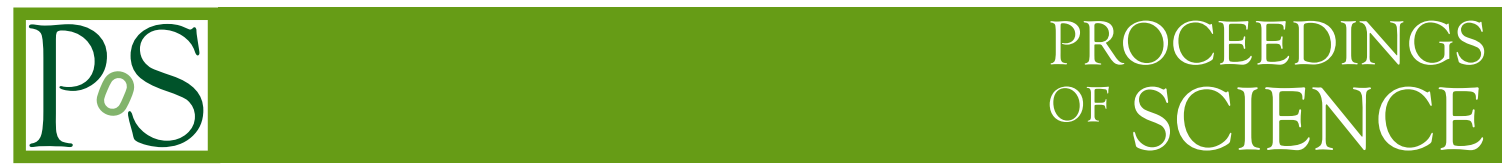

\title{
Simulation of Silicon Photomultipliers
}

\author{
Patrick ECKERT*, Rainer STAMEN, Hans-Christian SCHULTZ-COULON \\ Ruprecht-Karls-Universitaet Heidelberg (DE) \\ E-mail: patrick.eckertakip.uni-heidelberg.de
}

\begin{abstract}
We present a software framework for the simulation of Silicon Photomultipliers (SiPM). The simulation provides a detailed model of the SiPM response to arbitrary light pulses, depending on the basic SiPM characteristics which have to be determined by measurements. The simulation was validated for a S10362-11-100C Hamamatsu MPPC in the whole dynamic range and was used to study the contribution of different noise sources to the response and photon-counting resolution.
\end{abstract}

International Workshop on New Photon-detectors

June 13-15, 2012

LAL Orsay, France

${ }^{*}$ Speaker. 


\section{Introduction}

Silicon Photomultipliers (SiPM) are solid state photo-detectors with a wide range of applications in experimental particle physics, astrophysics and medical imaging [1, 2, 3]. The sensors consist out of an array of typically several hundred up to several thousand pixels per $1 \mathrm{~mm}^{2}$, which are connected to a common output. Each pixel is an avalanche photodiode (APD), which is operated above the breakdown voltage (Geiger mode); hence the signal of a single pixel is independent of the number of incident photons. The photon-counting capability is achieved by the segmentation of the sensor into pixels, thus the number of fired pixels is related to the number of incident photons.

The key features of SiPMs are a very compact size, insensitivity to magnetic fields, high gain and photon detection efficiency, as well as excellent single photon resolution and time resolution. However, there are also several sources of noise, like optical cross-talk between pixels, after-pulsing and a high dark-rate (typ. $100-1000 \mathrm{kHz}$ ). Furthermore, a pixel has a typical recovery time during which the signal caused by another incident photon on this pixel is reduced. Together with the limited number of pixels, this leads to a non-linear response of the sensor when the number of incident photons is of the order of the total number of pixels of the device.

Due to the complicated noise structure and the saturation behaviour at high light intensities, the response of SiPMs is quite complex compared to other photosensors. In order to obtain a detailed model of the SiPM response, the simulation framework $\mathrm{GosSiP}^{1}$ was developed which will be described in the following sections. A more detailed description can be found in [4].

\section{SiPM simulation}

Earlier simulation studies presented in $[5,6]$ already show a good description of the SiPM response for low light intensities. The simulation presented here was validated in the whole dynamic range, including the non-linear saturation regime at high light intensities, and thus complements previous studies. Furthermore, this simulation framework offers a more general tool which can be used to describe arbitrary sensors and operating conditions.

The input parameters of the simulation are the basic SiPM parameters: photon detection efficiency (PDE), gain, excess noise, dark-rate, cross-talk probability, after-pulse probabilities and after-pulse time constants, recovery time, number of pixels, the pixel arrangement and the single pixel pulse shape. These parameters have to be determined in characterisation measurements. A detailed description of the characterisation can be found in [7].

The simulation procedure starts off with the information about the spatial and time distribution of the incident photons. This information is generated by a basic simulation of a light source, where the photon distribution can be adjusted. Alternatively, external simulation software (e.g. Geant4) can be used to generate the photon information. In the next step, each photon can trigger a pixel signal with a certain probability given by the PDE. In addition, random pixel signals are generated in order to account for the dark-rate. Each pixel signal can trigger a signal in a neighbouring pixel, according to the cross-talk probability. Furthermore, a pixel signal can trigger a delayed signal in the same pixel as given by the after-pulse probability and time constant. In order to account for

\footnotetext{
${ }^{1}$ www.kip.uni-heidelberg.de/hep-detektoren/gossip
} 


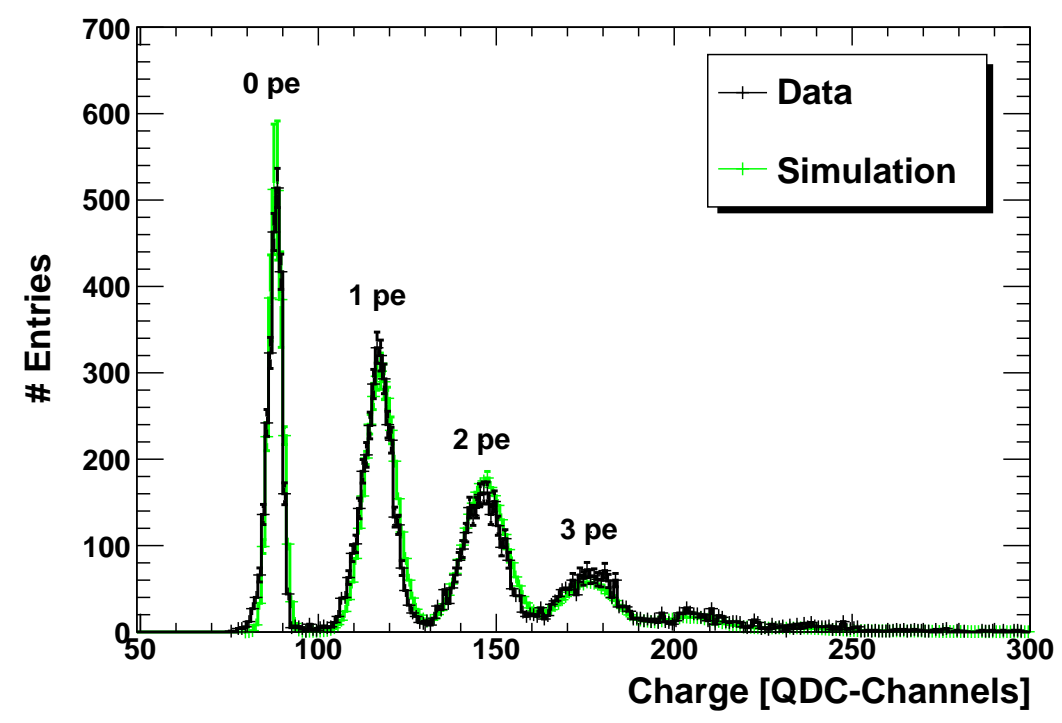

Figure 1: Single photo-electron spectrum for $0.5 \mathrm{~V}$ over-voltage. Each peak corresponds to a certain number of photo-electrons (pe).

recovery effects, the pixel signals have to be processed chronologically, since the pixel response depends on the time interval to the previous signal in the pixel. The pixel recovery is modelled by adjusting the parameters describing the pixel response (e.g. gain, PDE, ...) as a function of this time interval. The overall signal waveform and charge is composed by the sum of the individual pixel signals. The resulting signal can optionally be processed by a simplified simulation of the data acquisition which models common characterisation measurements.

\section{Response curve $\&$ resolution}

It is expected that the simulation provides a good model of the SiPM response for low light intensities, since the input parameters are determined from the single photon response $\mathrm{e}^{2}$ of the sensor. This is confirmed in [4] and was also shown in [5], where a similar simulation concept based on the chronological processing of pixel signals is used. However, for a complete validation it is essential to also test the simulation model in the high intensity saturation regime, where the recovery effects significantly influence the SiPM response. For the simulation presented here, this was done for a 100 pixel Hamamamtsu MPPC ${ }^{3}$ by measuring the SiPM response as a function of the incident light intensity and comparing the data to the simulation results.

The SiPM response is measured by illuminating the sensor with a pulsed laser diode in combination with a diffuser, which is used to achieve a homogeneous illumination of the sensor surface. The duration of the light pulse was estimated to be $1.0 \pm 0.5 \mathrm{~ns}$ which is well below the pixel recovery time of the sensor. A beam splitter evenly distributes the light to the SiPM and a calibrated PIN diode which serves as a reference sensor. For each light pulse, the signal charge is measured

\footnotetext{
${ }^{2}$ Response at very low light levels where the number of pixel signals can clearly be resolved.

${ }^{3} \mathrm{~S} 10362-11-100 \mathrm{C}$
} 

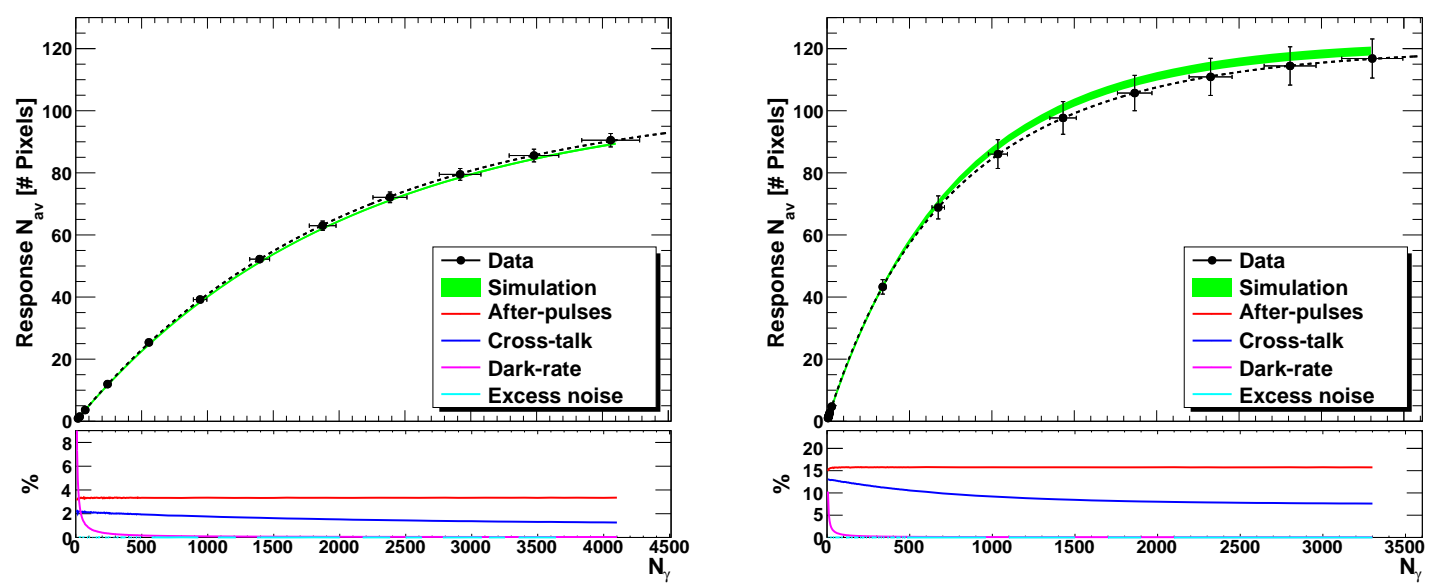

Figure 2: Top: SiPM response curve for low (left) and high (right) operation voltage. Bottom: Contributions from different noise sources.
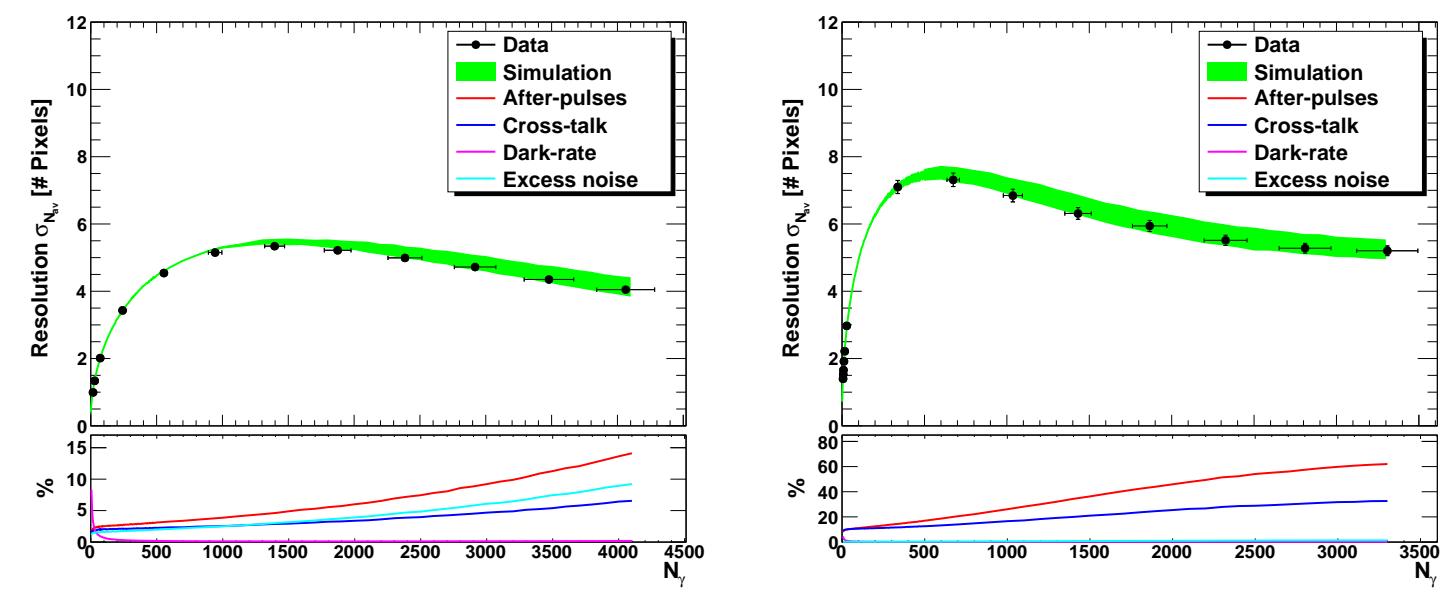

Figure 3: Top: RMS of the SiPM signal for low (left) and high (right) operation voltage. Bottom: Contributions from different noise sources.
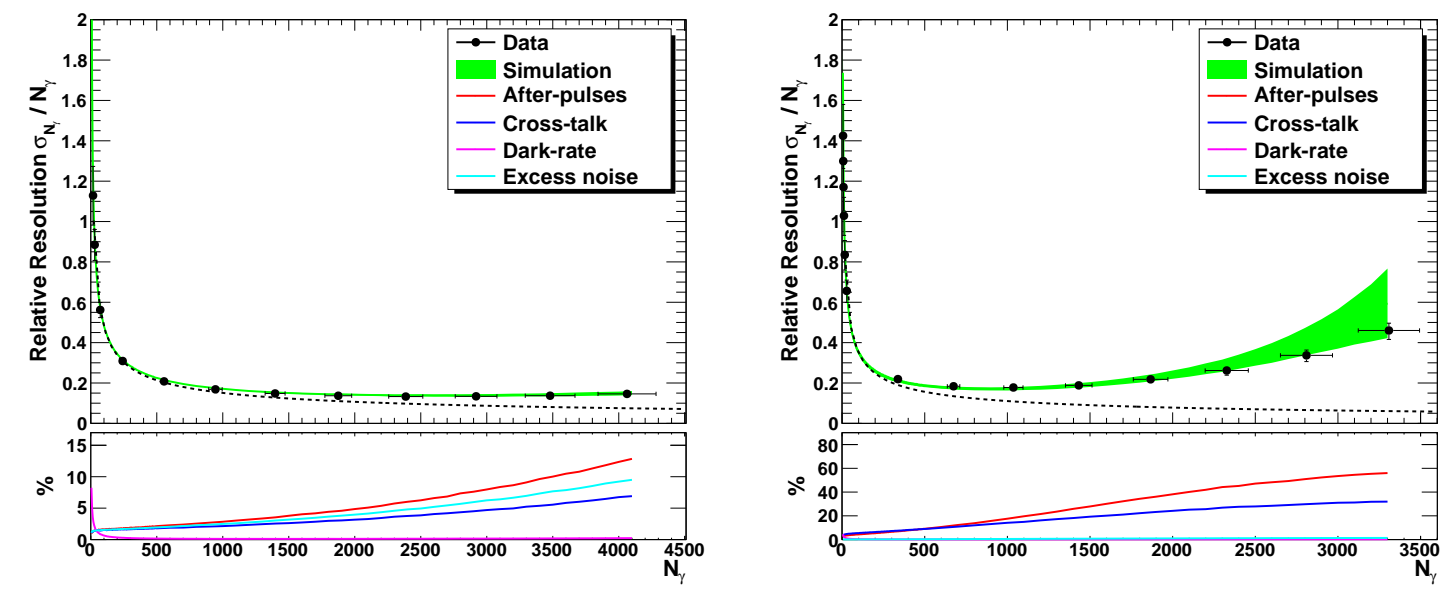

Figure 4: Top: Photon-counting resolution for low (left) and high (right) operation voltage. Bottom: Contributions from different noise sources. 
using a $\mathrm{QDC}^{4}$ module. A resulting charge spectrum for low light intensities is shown in figure 1. The individual peaks reflect the number of fired pixels, where the first peak corresponds to the charge of no fired pixel $Q_{\text {ped }}$ (pedestal), the second peak corresponds to the charge of one fired pixel $Q_{1 p e}$, and so on. It can be seen, that the spectrum is well described by the simulation. In the following, the SiPM response $N_{a v}$ is defined as the mean value of the charge spectrum $Q$ in units of fired pixels:

$$
N_{a v}=\frac{Q-Q_{p e d}}{Q_{1 p e}-Q_{p e d}} .
$$

The resolution is defined by the RMS of the spectrum in units of fired pixels.

Figure 2, 3 and 4 show the response and resolution as a function of the light intensity for low $(0.5 \mathrm{~V}$ over-voltage $)$ and high $(1.0 \mathrm{~V}$ over-voltage) operation voltage, which corresponds to a low and high noise level. The measured response and resolution is well reproduced by the simulation within the systematic uncertainties of the data points, and the uncertainty in the simulation which originates from the uncertainties in the input parameters. This demonstrates that the simulation provides an excellent model of the SiPM response in the whole dynamic range, an especially shows the validity of the recovery model which acts on all SiPM parameters.

From the simulation, the contributions from different noise sources to the signal and resolution was determined (see bottom of figures 2, 3, 4). For low light intensities, where the response is approximately linear, the contribution from noise to the resolution is relatively small; the resolution is dominated by statistical fluctuations in the number of detected photons due to a limited PDE. However, for high light intensities, cross-talk and after-pulsing significantly contribute to the resolution, especially in the case of high operation voltage, where the cross-talk and after-pulse probability is high.

\section{Summary}

In this proceeding a framework for the simulation of SiPMs is presented which provides a detailed model of the SiPM response and allows to model arbitrary sensors and operation conditions. Tests of the simulation for a 100 pixel sensor show an excellent agreement with measurement data in the whole dynamic range. The simulation was used to study the contribution of different noise sources to the response and resolution of the tested sensor. In the future, the simulation studies will be expanded to other SiPM devices.

\section{References}

[1] C. Adloff et al. [CALICE Collaboration], Construction and Commissioning of the CALICE Analog Hadron Calorimeter Prototype JINST 5 (2010) P05004

[2] A. Biland et al., First detection of air shower Cherenkov light by Geigermode-Avalanche Photodiodes, Nucl. Instrum. and Meth. A 595 (2008) 165

[3] S. Moehrs et al., A detector head design for small-animal PET with silicon photomultipliers (SiPM), Phys. Med. Biol. 51 (2006) 1113

\footnotetext{
${ }^{4}$ Charge-to-digital converter; LeCroy Model 2249; $50 \Omega$ input impedance.
} 
[4] P. Eckert et al., Study of the response and photon-counting resolution of silicon photomultipliers using a generic simulation framework, JINST 7 (2012) P08011

[5] A. Vacheret et al., Characterization and simulation of the response of Multi-Pixel Photon Counters to low light levels, Nucl. Instrum. and Meth. A 656 (2011) 69

[6] S. Sanchez Majos et al., Characterisation of radiation damage in silicon photomultipliers with a Monte Carlo model, Nucl. Instrum. and Meth. A 594 (2008) 351

[7] P. Eckert et al., Characterisation studies of silicon photomultipliers, Nucl. Instrum. and Meth. A 620 (2010) 217 